Published in final edited form as:

Urology. 2019 May ; 127: 19-23. doi:10.1016/j.urology.2019.02.024.

\title{
THE ASSOCIATION BETWEEN RACE AND FRAILTY IN OLDER ADULTS PRESENTING TO A NON-ONCOLOGIC UROLOGY PRACTICE
}

\author{
Samuel L. Washington III, MD ${ }^{1}$, Sima P. Porten, MD MPH ${ }^{1}$, Kathryn Quanstrom ${ }^{1}$, Chengshi \\ Jin $^{1}$, Mark Bridge ${ }^{1}$, Emily Finlayson, MD ${ }^{2,3}$, Louise C. Walter, MD², and Anne M. Suskind, \\ MD MS ${ }^{1}$ \\ ${ }^{1}$ Department of Urology, University of California, San Francisco \\ ${ }^{2}$ Division of Geriatrics, University of California, San Francisco and San Francisco VA Medical \\ Center \\ ${ }^{3}$ Division of General Surgery, University of California, San Francisco
}

\begin{abstract}
Objective: To explore whether there is an association between nonwhite race and frailty among older adults presenting to an academic non-oncologic urology practice.

Materials and Methods: This is a prospective study of individuals ages $\ 65$ years presenting to a non-oncologic urology practice between December 2015 and November 2016. All individuals had a Timed Up and Go Test (TUGT, where a slower TUGT time of $\geq 15$ seconds is suggestive of frailty. TUGT times, race (white vs nonwhite), and other clinical data were extracted from the electronic medical record using direct queries. Multivariable logistic regression was used to identify the association between race and slower TUGT times while adjusting for age, gender, number of medications, body mass index, and number of urologic diagnoses.
\end{abstract}

Results: Among the 1,715 individuals in our cohort, $33.9 \%$ were of nonwhite race and $15.3 \%$ had TUGT $\geq 15$ seconds. A higher percentage of nonwhite individuals had TUGT times $\geq 15$ seconds compared to white individuals ( $23.6 \%$ vs $11.1 \%$, p $<0.01$ ). TUGT times $\geq 15$ seconds were significantly associated with nonwhite race after adjusting for clinical factors (adjusted OR 2.5, 95\% CI 1.8-3.3).

Conclusions: Among older adults presenting to an academic non-oncologic urology practice, nonwhite race was associated with increased odds of frailty. A greater understanding of the relationship between race and frailty is needed to better address the needs of this vulnerable population.

Corresponding author: Samuel L. Washington III, MD, University of California, San Francisco, Department of Urology, Box 1695, San Francisco, California 94143-1695, Samuel.washington@ucsf.edu, Phone: 415-885-7748.

Publisher's Disclaimer: This is a PDF file of an unedited manuscript that has been accepted for publication. As a service to our customers we are providing this early version of the manuscript. The manuscript will undergo copyediting, typesetting, and review of the resulting proof before it is published in its final citable form. Please note that during the production process errors may be discovered which could affect the content, and all legal disclaimers that apply to the journal pertain. 


\section{Keywords}

Humans; Frailty; Prospective Studies; Risk Factors; Vulnerable Populations

\section{INTRODUCTION}

The majority of urologic procedures are performed in patients ages 65 and older, $20 \%$ of whom are considered to be frail. ${ }^{1-3}$ Frailty, a measure of heightened susceptibility to poor health outcomes in response to stressors, has been well documented to be independently associated with poor urologic postoperative outcomes including higher rates of complications, prolonged length of hospital stay, and higher rates of discharge to skilled or assisted nursing facilities. ${ }^{2-4}$

While measuring frailty allows clinicians to account for important non-disease specific characteristics, it does not take into account other essential factors also known to be associated with poor health outcomes such as race. Prior work has demonstrated that nonwhite race is independently associated with increased risk of in-hospital mortality, postoperative infections, and urinary, gastrointestinal, and cardiovascular complications. ${ }^{5}$ Race serves as a proxy for non-biological influences, incorporating more nuanced components such as socioeconomic factors and other social determinants which may negatively impact health. ${ }^{6}$ While both race and frailty are each independent and dynamic factors with known associations with poor health outcomes, little is known about the association between the two among patients presenting for non-oncologic urologic care.

In order to address this knowledge gap, we used data from the University of California, San Francisco Geriatric Urology Database (UCSF GUD). This is a prospective database inclusive of all individuals ages 65 and older presenting to our academic non-oncologic urology practice and includes the Timed Up and Go Test (TUGT) as a measure of frailty. Characterizing the interaction between race and frailty may elucidate potential targets for intervention and provide more focused methods to reduce disparities in vulnerable populations. Findings from this study will improve our understanding of the relationship between frailty and race, laying the groundwork for future improvements in urologic outcomes.

\section{MATERIALS AND METHODS}

\section{Patients and Database}

This study uses data from the UCSF GUD from December 2015 to November 2016. This is an Institutional Review Board approved database that prospectively collects data on all adults ages 65 and older presenting to our academic non-oncologic urology clinical practice. Data are regularly extracted from the electronic medical record using extract, transform and load sequences via the clinical data reporting database (Clarity) and data mart (Cogito). Details regarding data extraction have been previously published. ${ }^{7,8}$ 


\section{Outcomes}

The UCSF GUD is a unique urologic database in that it prospectively incorporates the TUGT, a validated proxy measure of frailty. ${ }^{7,9}$ The TUGT is administered to all individuals ages 65 and older presenting to our academic non-oncologic urology practice for a new patient visit, return patient visit or office-based procedure. It is administered at the beginning of the visit along with the vital signs. Patients are informed, with the use of an interpreter if needed, that they are doing a timed test as part of their vitals which gives a sense of their physical fitness. The TUGT consists of the time needed for a person to rise from a seated position in a chair, walk three meters to a mark on the floor, turn around and walk back to the chair, then sit down. This measure includes the assimilation of many different tasks, including understanding and following directions, walking speed, balance, and core strength. Based on the time it takes the individual to complete this task, the TUGT categorizes an individual as "fast" ( $\leq 10$ seconds), "intermediate" (11-14 seconds), or "slow" ( $\geq 15$ seconds), corresponding to "not frail", "intermediately frail" or "frail". ${ }^{9}$ Individuals who are non-ambulatory (i.e., in a wheelchair or gurney) are unable to perform the TUGT and are excluded from the analyses.

\section{Covariates}

Race was abstracted from the UCSF GUD and categorized as either "white" or "nonwhite" due to limited sample sizes within each self-reported racial group. Nonwhite race was inclusive of Asian, Black/African American, Native Hawaiian/Pacific Islander, American Indian/Alaska Native, and other. Race was self-reported by the patient at time of evaluation. "Other" was assigned if the patient declined to provide race information or the patient was unavailable to provide the information at the time of enrollment. Additional characteristics including age $(65-70,71-75,76-80,>80)$, gender, number of medications, and urologic diagnoses (based on categories previously published) were abstracted from the medical record. ${ }^{7}$ Body mass index (BMI) was included as an additional measure of health and comorbidity. In concordance with prior work number of medications was used as a proxy for comorbidity, which could not be accurately abstracted from the medical record. ${ }^{7,8}$

\section{Statistical analysis}

Summary characteristics were presented as means with standard deviations or as numbers with percentages, where appropriate. Characteristics were compared using t-tests or Chi square tests with two-sided $\mathrm{p}$ values. Multivariable logistic regression was used to identify factors associated with TUGT $\geq 15$ seconds, a surrogate measure of frailty, as the dependent variable. Independent variables included age, gender, race, number of medications, and number of urologic diagnoses. A two-way linear prediction plot was generated to graphically represent the univariable linear regression model between mean TUGT times and patient age. Results were stratified by race and displayed with $95 \%$ confidence intervals. A sensitivity analysis was performed using all self-reported race categories to evaluate the impact of the ,other " race category on the estimates for the association between race and TUGT scores 215 . A second sensitivity analysis excluded the „other " race category to to evaluate the impact of the ,other" race category on the estimates for the association between race and TUGT scores $\geq 15$. In both analyses, race groups including Asian, Black/African 
American, and American Indian/Alaska Native remained significantly associated with TUGT scores $\geq 15$ (Supplemental Tables 1 ). A p value $<0.05$ was statistically significant. Analyses were performed using STATA 14.3 software (College Station, Texas).

\section{RESULTS}

There were a total of 1,715 individuals ages $\searrow 65$ years with complete data during our study period. Nonwhite individuals comprised $33.9 \%$ of the cohort, $40.3 \%$ of whom self-identified as Asian, $13.3 \%$ as Black/African American, and 3.4\% as Native Hawaiian/Pacific Islander. Nonwhite older individuals tended to be slightly older ( $74.1 \pm 6.91$ years compared to 73 \pm 6.2 years, $\mathrm{p}<0.01$ ), had a higher percentage of females ( $27.5 \%$ compared to $22.0 \%$, $\mathrm{p}=0.01)$, and slower average TUGT times $(13.2 \pm 8.4$ versus $10.5 \pm 4.6$ seconds, $\mathrm{p}<0.01)$ with a higher percentage having "slow" TUGT times (23.6\% compared to $11.1 \%, \mathrm{p}<0.01$ ). The total number of medications and urologic diagnoses did not significantly differ between groups ( $\mathrm{p}=0.2$ and $\mathrm{p}=0.1$, respectively).

Results of the univariable and multivariable logistic regression models showing factors associated with slow TUGT times ( $\geq 15$ seconds) are shown in Table 2. In the multivariable logistic regression, nonwhite race was statistically significantly associated with TUGT times $\geq 15$ seconds (OR 2.5, 95\% CI 1.8-3.3, p<0.001) after adjusting for age, gender, number of medications, BMI, and number of urologic diagnoses. Older age, female gender, BMI, number of medications and number of urologic diagnoses also had significant associations with TUGT times $\geq 15$ seconds. When stratified by race, mean TUGT scores were significantly higher at all ages for nonwhite individuals compared to white individuals $(\mathrm{p}<0.01)$ (figure).

\section{DISCUSSION}

Our study demonstrated that nonwhite individuals have slower TUGT times compared to white individuals (23.6\% compared to $11.1 \%$ with TUGT $\geq 15$ seconds, respectively). Nonwhite race was significantly associated with slower TUGT times (OR 2.5, 95\% CI 1.83.3), even after adjusting for other factors such as age, gender, BMI, number of medications, and number of urologic diseases. Our findings also demonstrated that nonwhite individuals have slower TUGT times compared to white individuals across all age groups studied among individuals presenting for care at a non-oncologic urology practice and that this difference become more pronounced with older age.

This is the first study to investigate the association between race and frailty, as measured by slower TUGT times, in a non-oncologic cohort. Both race and TUGT times serve as surrogate variables for various unmeasurable factors. While there is no current consensus on how best to measure frailty, TUGT times have been shown to be closely associated with frailty and age. ${ }^{7}$ Independent of age, slower TUGT times/frailty are thought to reflect factors such as impaired mobility, a reduced physiologic reserve to respond to stressors, and accrued deficits in multiple organ systems over time. ${ }^{10-12}$ Race has been shown to be associated with differences in socioeconomic status, access to and receipt of healthcare, and reflects the differences in lifetime exposures due to social environment. ${ }^{13,14}$ Although independent from 
one another, both race and frailty reflect the consequences of complex interactions between various genetic, physiologic, and environmental influences over one's lifespan.

Our findings demonstrate the association between race, a proxy for non-biological influences, and frailty, yet little is known of the character of this relationship. In a study of community-dwelling individuals aged 65 years or older, education, decreasing social resources, and poor quality of life were found to be associated with increased frailty. ${ }^{15}$ These findings suggest the impact of frailty may be influenced by social determinants known to be associated with racial disparities in outcomes. ${ }^{14}$ While identification of these interactions is beyond the scope of this study, our findings support the hypothesis that these independent concepts of race, as a social construct, and frailty may in fact harbor a commonality which has not previously been examined. Further investigation with focus on the granular differences in social determinants and its interconnectivity with frailty may lay the groundwork for holistic approaches to reducing disparities in vulnerable populations.

Our study shows an association between race and slower TUGT times persists across the age spectrum, which is congruent with prior literature. A previous study used the US National Health and Nutrition Examination Survey to explore the relationship between age and frailty over one's lifespan. The authors measured frailty using the Frailty index and compared scores of those younger than 65 years to those older than 65 years. They demonstrated a significant correlation between age and frailty with both age groups. ${ }^{16}$ Similar to this study, another used TUGT times as a proxy for frailty but instead focused on the association with postoperative outcomes for patients undergoing elective cardiac and cardiac operations. Within both groups they noted a significant difference in TUGT times as age increased. ${ }^{9}$ Our findings not only support the finding that frailty increases with age but also suggest that the magnitude of this relationship may be influenced by race, independent of age.

Our study should be considered with certain limitations in mind. First, our findings represent the experience of a single academic non-oncologic urology practice, which may affect the generalizability of the findings. Because our center is a tertiary referral center, our study cohort may represent sicker or more frail individuals than those seen in other practices. However, our patient population is likely similar to those in other academic institutions and novel in its evaluation of frailty in a non-oncologic urology practice. Further studies among other types of urology practices are warranted in order to assess the generalizability of our findings. Second, our study uses one measure of frailty, the TUGT, which has been shown to be susceptible to false positives of frailty. ${ }^{17}$ Unfortunately, there is no one perfect measure of frailty that is agreed upon by all clinicians and researchers. We feel that the TUGT is an ideal measure for this purpose, as it is a quick and easy test to administer during clinical care that has demonstrated association with postoperative complications and 1-year mortality among surgical populations. ${ }^{9}$ Lastly, additional detail of socioeconomic factors such as education, household income, health literacy, allostatic stress, and social network of study participants may provide more nuanced information about how these factors differ between groups and impact one's risk of frailty. Further evaluation with detailed data on socioeconomic factors, lifestyle habits such as smoking, and social determinants of health may offer more nuanced information on the interactions between race, social determinants, and frailty risk. Lastly, the design of our study limits our ability to comment on causal 
pathways between race and frailty, limiting our investigation to identifying significant associations between these factors. Despite this shortcoming, we strongly feel that this is an excellent starting point to make these observations and future research is needed to further disentangle and explore these findings.

\section{CONCLUSIONS}

Among older adults presenting to an academic non-oncologic urology practice, nonwhite race is associated with increased frailty across all age groups studied. Our findings suggest a relationship between social determinants and unmeasured physiologic factors that has not been previously described. Understanding the influence of the interaction between race and frailty may provide future targets for intervention and focused methods to reduce disparities in vulnerable populations.

\section{Supplementary Material}

Refer to Web version on PubMed Central for supplementary material.

\section{Source of Funding:}

NIDDK K12 DK83021-09; K12 Urologic Research (KURe) Career Development Program, UCSF Clinical \& Translational Science Institute grant \#UL1 TR000004

\section{REFERENCES}

1. Drach GW and Griebling TL: Geriatric Urology. Journal of the American Geriatrics Society 2003; 51: S355-S358. [PubMed: 12823668]

2. Suskind AM, Walter LC, Jin C, et al.: Impact of frailty on complications in patients undergoing common urological procedures: a study from the American College of Surgeons National Surgical Quality Improvement database. BJU Int. 2016; 117: 836-842. [PubMed: 26691588]

3. Makary MA, Segev DL, Pronovost PJ, et al.: Frailty as a Predictor of Surgical Outcomes in Older Patients. ACS 2010; 210: 901-908.

4. Suskind AM, Jin C, Cooperberg MR, et al.: Preoperative Frailty Is Associated With Discharge to Skilled or Assisted Living Facilities After Urologic Procedures of Varying Complexity. Urology 2016; 97: 25-32. [PubMed: 27392651]

5. Akinyemiju T, Meng Q and Vin-Raviv N: Race/ethnicity and socio-economic differences in colorectal cancer surgery outcomes: analysis of the nationwide inpatient sample. BMC Cancer 2016: 1-10.

6. Solar O and Irwin A: A conceptual framework for action on the social determinants of health. 2010: $1-78$.

7. Pangilinan J, Quanstrom K, Bridge M, et al.: The Timed Up and Go Test as a measure of frailty in urologic practice. Urology 2016: 1-22.

8. Suskind AM, Quanstrom K, Zhao S, et al.: Overactive bladder is strongly associated with frailty in older individuals. Urology 2017: 1-36.

9. Robinson TN, Wu DS, Sauaia A, et al.: Slower Walking Speed Forecasts Increased Postoperative Morbidity and 1-Year Mortality Across Surgical Specialties. Annals of Surgery 2013: 1-19.

10. Bellal J and Faisal JS: The Mobility and Impact of Frailty in the Intensive Care Unit. Surgical Clinics of NA 2017; 97: 1199-1213.

11. Ruiz M, Cefalu C and Reske T: Frailty syndrome in geriatric medicine. Am. J. Med. Sci 2012; 344: 395-398. [PubMed: 22739566] 
12. Fried LP, Tangen CM, Walston J, et al.: Frailty in Older AdultsEvidence for a Phenotype. J Gerontol A Biol Sci Med Sci 2001; 56: M146-M157. [PubMed: 11253156]

13. Williams DR: Race, Socioeconomic Status, and Health The Added Effects of Racism and Discrimination. Annals of the New York Academy of Sciences 2006; 896: 173-188.

14. Williams DR, Priest N and Anderson NB: Understanding associations among race, socioeconomic status, and health: Patterns and prospects. Health Psychology 2016; 35: 407-411. [PubMed: 27018733]

15. de Labra C, Maseda A, Lorenzo-López L, et al.: Social factors and quality of life aspects on frailty syndrome in community-dwelling older adults: the VERISAÚDE study. BMC Geriatr 2018; 18: 66. [PubMed: 29514599]

16. Kane AE, Gregson E, Theou O, et al.: The association between frailty, the metabolic syndrome, and mortality over the lifespan. 2017: 1-9.

17. Clegg A, Rogers L and Young J: Diagnostic test accuracy of simple instruments for identifying frailty in community-dwelling older people: a systematic review. Age Ageing 2015; 44: 148-152. [PubMed: 25355618] 

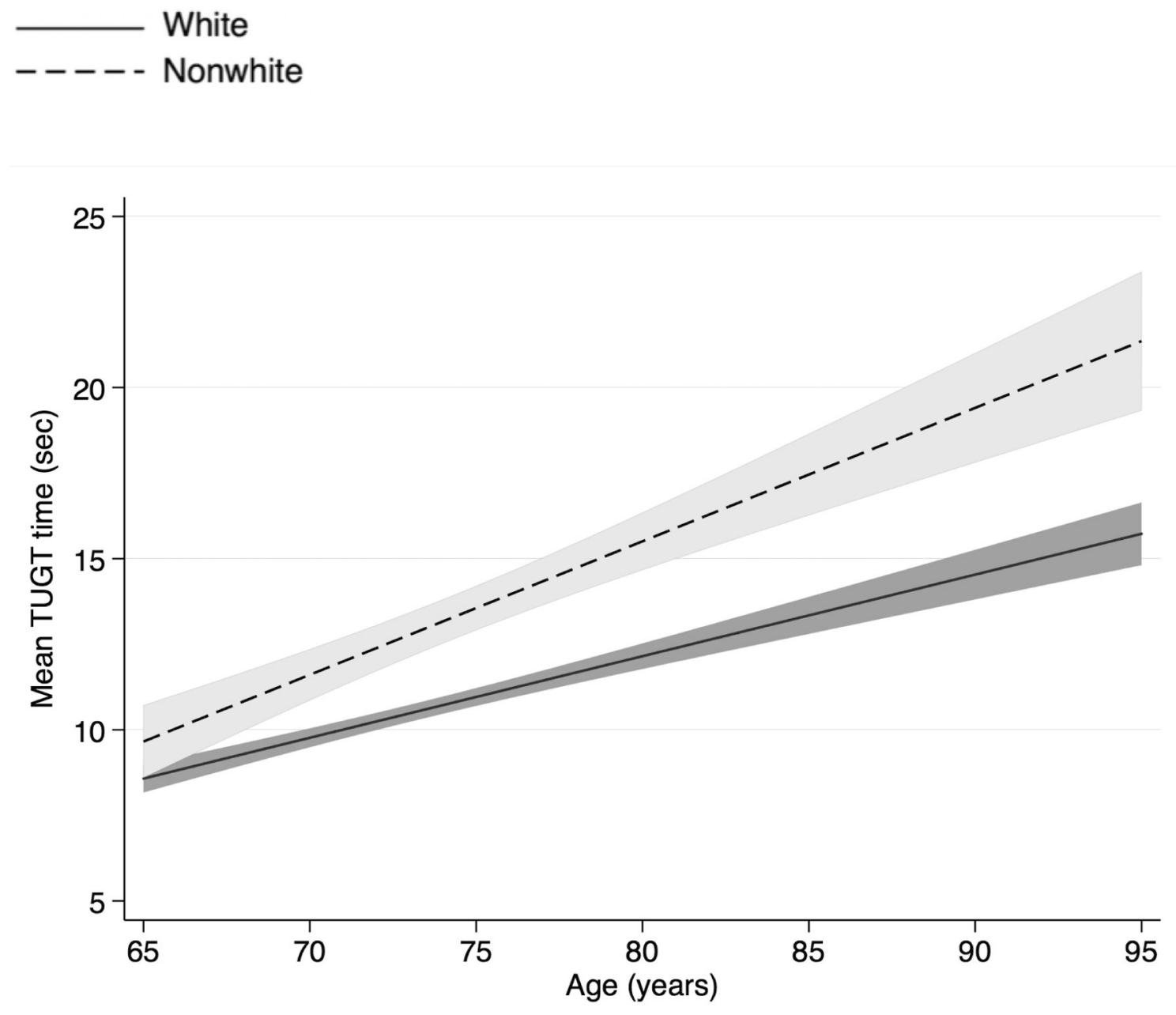

Figure:

Relationship between mean TUGT time and age, stratified by race 
Table 1.

Demographic characteristics for cohort overall and stratified by race $(n=1715)$

\begin{tabular}{|c|c|c|c|c|}
\hline Characteristic & $\begin{array}{l}\text { Overall Cohort } \\
\quad(N=1715)\end{array}$ & $\begin{array}{c}\text { White } \\
(\mathrm{N}=1134)\end{array}$ & $\begin{array}{l}\text { Non-White } \\
(\mathbf{N}=581)\end{array}$ & P-Value \\
\hline Age (Mean \pm SD) & $73.4 \pm 6.5$ & $73 \pm 6.2$ & $74.1 \pm 6.9$ & $<0.01$ \\
\hline \multicolumn{5}{|l|}{ Age group (in years), n (\%) } \\
\hline $65-70$ & $689(40.2 \%)$ & $467(41.2 \%)$ & $222(38.2 \%)$ & $<0.01$ \\
\hline $71-75$ & $493(28.7 \%)$ & $346(30.5 \%)$ & $147(25.3 \%)$ & \\
\hline $76-80$ & $272(15.9 \%)$ & $176(15.5 \%)$ & $96(16.5 \%)$ & \\
\hline$>80$ & $261(15.2 \%)$ & $145(12.8 \%)$ & $116(20.0 \%)$ & \\
\hline \multicolumn{5}{|l|}{ Gender, n $(\%)$} \\
\hline Male & $1305(76.1 \%)$ & $884(78.0 \%)$ & $421(72.5 \%)$ & 0.01 \\
\hline Female & $410(23.9 \%)$ & $250(22.0 \%)$ & $160(27.5 \%)$ & \\
\hline \multicolumn{5}{|l|}{ Race, n (\%) } \\
\hline White/Caucasian & $1134(66.1 \%)$ & $1134(100.0 \%)$ & -- & $<0.01$ \\
\hline Asian & $234(13.6 \%)$ & -- & $234(40.3 \%)$ & \\
\hline Black/African American & $77(4.5 \%)$ & -- & $77(13.3 \%)$ & \\
\hline Native Hawaiian/Pacific Islander & $20(1.2 \%)$ & -- & $20(3.4 \%)$ & \\
\hline American Indian/Alaska Native & $7(0.4 \%)$ & -- & $7(1.2 \%)$ & \\
\hline Other & $242(14.1 \%)$ & -- & $243(41.9 \%)$ & \\
\hline Total number of meds per person (Mean \pm SD) & $7.7 \pm 5.5$ & $7.6 \pm 5.4$ & $7.9 \pm 5.5$ & 0.2 \\
\hline BMI $($ Mean \pm SD $)$ & $27.1 \pm 4.9$ & $27.3 \pm 4.76$ & $26.7 \pm 5.1$ & $<0.01$ \\
\hline Total number of urologic diagnoses per person (Mean $\pm \mathrm{SD})$ & $1.3 \pm 0.8$ & $1.3 \pm 0.9$ & $1.4 \pm 0.8$ & 0.1 \\
\hline Stones/Ureteral obstruction/Ureteral stricture & $289(16.9 \%)$ & $200(17.6 \%)$ & $89(15.3 \%)$ & 0.2 \\
\hline Male general urology & $410(23.9 \%)$ & $305(26.9 \%)$ & $105(18.1 \%)$ & $<0.01$ \\
\hline LUTS/BPH/Retention/Nocturia & $530(30.9 \%)$ & $309(27.2 \%)$ & $221(38.0 \%)$ & $<0.01$ \\
\hline Urinary tract infection & $158(9.2 \%)$ & $101(8.9 \%)$ & $57(9.8 \%)$ & 0.5 \\
\hline Urinary incontinence & $209(12.2 \%)$ & $143(12.6 \%)$ & $66(11.4 \%)$ & 0.5 \\
\hline Urgency/Frequency/OAB & $261(15.2 \%)$ & $165(14.6 \%)$ & $96(16.5 \%)$ & 0.3 \\
\hline Male urethral disease & $137(8.0 \%)$ & $93(8.2 \%)$ & $44(7.6 \%)$ & 0.7 \\
\hline Neurogenic bladder & $65(3.8 \%)$ & $53(4.7 \%)$ & $12(2.1 \%)$ & $<0.01$ \\
\hline Hematuria & $172(10.0 \%)$ & $94(8.3 \%)$ & $78(13.4 \%)$ & $<0.01$ \\
\hline Female reconstruction & $34(2.0 \%)$ & $19(1.7 \%)$ & $15(2.6 \%)$ & 0.2 \\
\hline TUGT time in seconds (Mean \pm SD) & $11.4 \pm 6.3$ & $10.5 \pm 4.6$ & $13.2 \pm 8.5$ & $<0.01$ \\
\hline \multicolumn{5}{|l|}{ TUGT time category, $\mathrm{n}(\%)$} \\
\hline Fast ( $\leq 0$ seconds) & $963(56.2 \%)$ & $728(64.2 \%)$ & $235(40.5 \%)$ & $<0.01$ \\
\hline Intermediate (11-14 seconds) & $489(28.5 \%)$ & $280(24.7 \%)$ & $209(36.0 \%)$ & \\
\hline Slow ( $\geq 15$ seconds) & $263(15.3 \%)$ & $126(11.1 \%)$ & $137(23.6 \%)$ & \\
\hline
\end{tabular}


Table 2.

Logistic regression models predicting TUGT time $>15$ seconds

\begin{tabular}{lcccccccc}
\hline & & \multicolumn{3}{c}{ Univariable } & \multicolumn{4}{c}{ Multivariable } \\
Characteristic & Value & OR & $\mathbf{9 5 \%}$ CI & P-Value & OR & $\mathbf{9 5 \% ~ C I ~}$ & P-Value \\
Age group (in years) & $65-70$ & Ref & - & & Ref & - & \\
& $71-75$ & 1.9 & $1.3-2.9$ & $<0.01$ & 2.1 & $1.3-3.2$ & $<0.01$ \\
& $76-80$ & 5.0 & $3.3-7.6$ & $<0.01$ & 4.6 & $2.9-7.3$ & $<0.01$ \\
& $>80$ & 9.4 & $6.3-14.0$ & $<0.01$ & 9.9 & $6.3-15.5$ & $<0.01$ \\
Gender & Male & Ref & - & & Ref & - & \\
& Female & 2.4 & $1.9-3.2$ & $<0.01$ & 2.3 & $1.6-3.1$ & $<0.01$ \\
Race & White & Ref & - & & Ref & - & \\
& Nonwhite & 2.5 & $1.9-3.2$ & $<0.01$ & 2.5 & $1.8-3.3$ & $<0.01$ \\
Number of medications & & 1.1 & $1.0-1.1$ & $<0.01$ & 1.1 & $1.0-1.1$ & $<0.01$ \\
BMI (per 1-unit increase) & & 1.0 & $0.9-1.0$ & 0.4 & 1.0 & $1.0-1.1$ & 0.01 \\
Number of Urologic diagnoses per person & & 1.6 & $1.4-1.9$ & $<0.01$ & 1.4 & $1.2-1.7$ & $<0.01$ \\
\hline
\end{tabular}

